\title{
Proposal of Particle Swarm Optimization Methods with Nonlinear Dissipative Term
}

\author{
Hideki Murata Non-member (Keio Uiversity) \\ Keiichiro Yasuda Member (Tokyo Metropolitan University) \\ Eitaro Aiyoshi Member (Keio Uiversity)
}

Keywords: global optimization, particle swarm optimization, disspative dynamical system

\section{Introduction}

In this paper, we propose three types of new Particle Swarm Optimization (PSO) method. PSO, one of global optimization methods, is known for its high searching ability and easy implement. However, it might be difficult to find the global optimum for optimization problems which have a lot of decision variables and local optima. Proposed models improve searching ability through such cases. One is a model with the nonlinear dissipative term to prohibit the search point's velocity being zero. The others are models with the nonlinear dissipative term with the pbest or the gbest information to disturb the search around them.

\section{Proposed Models}

The dynamics of a continuous time PSO model for an object function $E: \boldsymbol{R}^{n} \rightarrow \boldsymbol{R}$ is written as the following equations.

$$
\begin{aligned}
& \frac{d^{2} \boldsymbol{x}^{p}(t)}{d t^{2}}+a \frac{d \boldsymbol{x}^{p}(t)}{d t}=c\left[c_{1}\left\{\boldsymbol{x}^{p}\left(T^{p}(t)\right)-\boldsymbol{x}^{p}\right\}\right. \\
& \left.+c_{2}\left\{\boldsymbol{x}^{Q(t)}\left(T^{o}(t)\right)-\boldsymbol{x}^{p}\right\}\right] \\
& \text {..................... (1a) } \\
& T^{p}(t)=\operatorname{argmin}\left\{E\left(\boldsymbol{x}^{p}(\tau)\right) \mid 0 \leq \tau \leq t\right\} \\
& \left(Q(t), T^{o}(t)\right)= \\
& \operatorname{argmin}\left\{E\left(\boldsymbol{x}^{q}(\tau)\right) \mid q=1, \cdots, P, 0 \leq \tau \leq t\right\}
\end{aligned}
$$$$
\ldots \ldots \ldots \ldots \ldots \ldots \ldots,(1 \mathrm{c})
$$

where $\boldsymbol{x}^{p}(t)$ shows the position of $p$-th searching point at $t$ . Now we change the linear dissipative term $a \frac{d \boldsymbol{x}^{p}(t)}{d t}$ into a nonlinear dissipative term which prohibits the search point's velocity being zero and introduce a state variable $\boldsymbol{v}^{p}$. Furthermore by making it discrete through Eular method, the following discrete time model is obtained.

$$
\begin{aligned}
& v_{i}^{p}(k+1)= \lambda\left(\boldsymbol{v}^{p}(k)\right) v_{i}^{p}(k) \\
&+c_{1} r_{1 i}\left\{x_{i}^{p}\left(l^{p}(k)\right)-x_{i}^{p}(k)\right\} \\
&+c_{2} r_{2 i}\left\{x_{i}^{Q(k)}\left(l^{o}(k)\right)-x_{i}^{p}(k)\right\} \cdots \cdots \\
& x_{i}^{p}(k+1)= x_{i}^{p}(k)+v_{i}^{p}(k+1) \cdots \cdots \cdots \cdots \cdots \\
& \lambda(\boldsymbol{v})=1-d_{1}+d_{1} d_{0} \exp \left(\frac{-\|\boldsymbol{v}\|}{d_{2} d_{0}}\right) \cdots \cdots \cdots \cdots \\
& l^{p}(k)=\underset{l}{\operatorname{argmin}}\left\{E\left(\boldsymbol{x}^{p}(l)\right) \mid l=0,1, \cdots, k\right\} \cdots \cdots \\
&\left(Q(k), l^{o}(k)\right)=\underset{(q, l)}{\operatorname{argmin}}\left\{E \left(\boldsymbol{x}^{q}(l) \mid q=1,2, \cdots, P,\right.\right. \\
&l=0,1, \cdots, k\} \cdots \cdots \cdots
\end{aligned}
$$

where $r_{i 1}, r_{i 2}$ is uniform random numbers in $[0,1]$. This model is called model.1. Other two proposed models are given the following nonlinear inertia weights $\lambda$.

\begin{tabular}{|c|c|c|c|c|c|c|c|}
\hline benchmark & nodel & $\mathrm{CR}$ & mean value & best value & wors t value & SD & \begin{tabular}{|l|} 
CPU.Tim \\
\end{tabular} \\
\hline \multirow{4}{*}{$\begin{array}{c}\text { Rastrigin } \\
(\mathrm{n}=12)\end{array}$} & $\begin{array}{l}\text { PSO-NXX } \\
\end{array}$ & $2 \%$ & 3.9109 & $\begin{array}{ll}0.0 \\
\end{array}$ & 9.94586 & 3.212463 & $\begin{array}{l}310.99 \\
\end{array}$ \\
\hline & nodel. $1\left(d_{0}(0)=4 \bowtie 0\right)$ & $56 \%$ & 0.606925 & 0.0 & 2.984877 & 0.591557 & 342.44 \\
\hline & nodel. $2\left(d_{0}(0)=3 \triangleright 0\right)$ & $96 \%$ & 0.039798 & 0.0 & 0.994959 & 0.038014 & 364.57 \\
\hline & nodel.3 $\left(d_{0}(0)=3 \triangleright 0\right)$ & $96 \%$ & 0.039798 & 0.0 & 0.994959 & 0.038014 & 365.33 \\
\hline \multirow{4}{*}{$\begin{array}{c}\text { Levy-No.5 } \\
(\mathrm{n}=50)\end{array}$} & PSO-KXI & $75 \%$ & 0.027089 & 0.0 & 0.370709 & 0.003283 & 2690.3 \\
\hline & nodel.1 $\left(d_{0}(0)=4 \bowtie 0\right)$ & $88 \%$ & 0.011082 & 0.0 & 0.184643 & 0.001090 & 2836.5 \\
\hline & nodel.2( $\left.d_{0}(0)=3 \triangleright 0\right)$ & $91 \%$ & 0.00431 & 0.0 & 0.061585 & 0.000247 & 2904.2 \\
\hline & nodel.3 $\left(d_{0}(0)=3 \bowtie 0\right)$ & $96 \%$ & 0.00431 & 0.0 & 0.123114 & 0.000323 & 2908.3 \\
\hline \multirow{4}{*}{$\begin{array}{c}\text { Grie wank } \\
(\mathrm{n}=6)\end{array}$} & $\begin{array}{l}\text { PSO-NWX } \\
\end{array}$ & $39 \%$ & 0.035641 & 0.0 & 0.114410 & 0.000960 & 201.58 \\
\hline & nodel.1 $\left(d_{0}(0)=8 \bowtie 0\right)$ & $77 \%$ & 0.012826 & 0.0 & 0.081540 & 0.000582 & 233.73 \\
\hline & nodel.2( $\left(d_{0}(0)=8 \bowtie 0\right)$ & $91 \%$ & 0.04335 & 0.0 & 0.065254 & 0.000197 & 250.05 \\
\hline & nodel.3 $\left(d_{0}(0)=8 \bowtie 0\right)$ & $95 \%$ & 0.002047 & 0.0 & 0.040495 & 0.000080 & 249.55 \\
\hline \multirow{4}{*}{$\begin{array}{c}\text { 2n-minima } \\
(\mathrm{n}=14)\end{array}$} & PSO-NWA & $51 \%$ & $\begin{array}{l}-1077.71 \\
\end{array}$ & $\begin{array}{l}-1096.65 \\
\end{array}$ & $\begin{array}{ll}-1011.83 \\
\end{array}$ & 480.511 & 268.48 \\
\hline & nodel.1 $\left(d_{0}(0)=18 \triangleright 0\right)$ & $56 \%$ & -1079.97 & -1096.65 & -1011.83 & 449.175 & 308.24 \\
\hline & nodel.2 $\left(d_{0}(0)=10 \bowtie 0\right)$ & $96 \%$ & -1095.52 & -1096.65 & -1068.38 & 30.6964 & 321.46 \\
\hline & nodel. $3\left(d_{0}(0)=10 \triangleright 0\right)$ & $92 \%$ & -1094.39 & -1096.65 & -1068.38 & 58.8349 & 319.85 \\
\hline
\end{tabular}

model.2:

$$
\begin{aligned}
& \lambda\left(\boldsymbol{x}^{p}\left(l^{p}(k)\right)-\boldsymbol{x}^{p}(k)\right)= \\
& \quad 1-d_{1}+d_{1} d_{0} \exp \left(\frac{-\left\|\boldsymbol{x}^{p}\left(l^{p}(k)\right)-\boldsymbol{x}^{p}(k)\right\|}{d_{2} d_{0}}\right)
\end{aligned}
$$

model.3:

$$
\begin{aligned}
& \lambda\left(\boldsymbol{x}^{Q(k)}\left(l^{o}(k)\right)-\boldsymbol{x}^{p}(k)\right)= \\
& \quad 1-d_{1}+d_{1} d_{0} \exp \left(\frac{-\left\|\boldsymbol{x}^{Q(k)}\left(l^{o}(k)\right)-\boldsymbol{x}^{p}(k)\right\|}{d_{2} d_{0}}\right)
\end{aligned}
$$

\section{Compuational Results}

The computational results for some benchmarks are shown in Table 1. The convergence rate to global minimum, the mean value, the best value, the worst value, and standard deviation of the value of gbest through 100 trials by each model with 20 particles are shown in Table 1.

Table 1. Comparison of searching ability

CR. Convergence Rate SD:Standard Deviation 


\title{
非線形散逸項を有する Particle Swarm Optimization 法の提案
}

\author{
非会員 村田 秀樹* 正 員 安田 恵一郎** \\ 正 員 相吉 英太郎*
}

\author{
Proposal of Particle Swarm Optimization Methods with Nonlinear Dissipative Term \\ Hideki Murata*, Non-member, Keiichiro Yasuda**, Member, Eitaro Aiyoshi*, Member
}

\begin{abstract}
Optimization methods based on meta-heuristics are proposed as a class of global optimization methods, by which the global minimum can be obtained without trapping in local minima. Particle swarm optimization(PSO), which is one of those methods, is known for its high search ability and easy implement. However, it might be difficult to find the global optimum for optimization problems which have a lot of decision variables and local optima. In this paper, we propose three types of new PSO to clear the weak point. One is a model with the nonlinear dissipative term intoroduced by Fujita, Yasuda and Yokoyama ${ }^{(4)}$ to prohibit the search point's velocity being zero. The others are models with the nonlinear dissipative term with the pbest or the gbest information to disturb the search around them.
\end{abstract}

キーワード：大域的最適化, Particle Swarm Optimization, 散逸力学系

Keywords: global optimization, particle swarm optimization, disspative dynamical system

\section{1. はじめに}

最適化問題は, ある決定変数に従属して決まる目的関数 の值を最大または最小にするような解，つまり最適解を求 める問題のことで, 制御系や信号処理システムの設計, 機 械構造物の設計, 電力系統などのエネルギーシステムの設 計には不可欠な問題である。とくに，目的関数が多峰性関 数の場合において, 局所的最適解への停留を回避して, 大 域的最適解を得る大域的最適化手法の開発が, この分野の 重要な研究テーマとなっている。最近では, メタヒューリス ティックスという概念に基づく大域的最適化手法の一つであ る, Particle Swarm Optimization ${ }^{(1) \sim(3)}$ (以下 PSO と略 する) が，そのアルゴリズムの簡便さや大域的探索性能の高 さから注目を集めているが, 目的関数の多峰性が強く, か つ変数次元が高い問題の場合には, 探索性能が劣化するこ とが指摘されている。そこで本論文では, PSO モデルの改 良により, 変数次元の高い問題に対しても, その大域的探 索性能をある程度維持できる新しい計算モデルを提案する。

\footnotetext{
*慶應義塾大学理工学部

干 223-8522 横浜市港北区日吉 3-14-1

Faculty of Science and Tcechnology, Keio University

3-14-1 Hiyoshi, Kouhoku-ku, Yokohama, 223-8522

** 東京都立大学大学院工学研究科

干 192-0397 東京都八王子市南大沢 1-1

Faculty of Engineering, Tokyo Metropolitan University

1-1 Minami Oosawa, Hachioji, Tokyo, 192-0397
}

ところで，PSO の計算モデルは，速度が等比数列形の線 形慣性系のモデルに, pbest と称する過去における各探索 点の最良位置への移流方向と, gbest と称する探索点群全体 の過去の最良位置への移流方向の畳み込みを外部入力とす る多点型離散力学系モデルである。そこで本論文では, 探 索点単独の慣性系モデルを非線形化することより, 探索点 自体のダイナミクスの機能を強化した上で, pbest や gbest の情報もその非線形要素に取り込むことにより, 結合型非 線形力学系として, その動特性を高機能化することを考え る。具体的には, (1) 探索点単独の非線形モデルとして, 速 度がゼロとなる停留状態を回避する負性抵抗を有する非線 形散逸モデル (4)，(2) 結合系としての特徵を生かすために, 負性抵抗の項に pbest や gbest の情報を加えることにより， それらに近接すると負性抵抗によってそれらから反発する ような非線形散逸モデル, の導入である。これらのモデル による多点探索により, 従前型の PSO では探索の進渉につ れて, pbest や gbestに収束して探索の多様性を喪失してし まう現象を防ぐことができ, 変数次元の高い問題に対して も，より良好な大域的探索性能を有するものと期待される。

\section{Particle Swarm Optimization(PSO)}

$\mathrm{PSO}^{(1) \sim(3)}$ では, Particle と呼ばれる複数の探索点（以 降個体と称する）が, それぞれの位置と速度を, 逐次更新 しながら解空間を探索する。目的関数 $E: \boldsymbol{R}^{n} \rightarrow \boldsymbol{R}^{1}$ に対 する無制約最適化問題 
$\min _{\boldsymbol{x}} E(\boldsymbol{x})$

を解くための, PSO の更新式は,

$$
\begin{aligned}
& v_{i}^{p}(k+1)=\lambda v_{i}^{p}(k)+c_{1} r_{1 i}\left\{x_{i}^{p}\left(l^{p}(k)\right)-x_{i}^{p}(k)\right\} \\
& +c_{2} r_{2 i}\left\{x_{i}^{Q(k)}\left(l^{o}(k)\right)-x_{i}^{p}(k)\right\} \\
& \text {................ (2a) } \\
& x_{i}^{p}(k+1)=x_{i}^{p}(k)+v_{i}^{p}(k+1) \\
& i=1,2, \cdots, n \quad p=1,2, \cdots, P \quad k=0,1,2, \cdots
\end{aligned}
$$

と書くことができる。ただし $, x_{i}^{p}(k), v_{i}^{p}(k)$ は $p$ 番目の個 体の時刻 $k$ に抢ける位置と速度の第 $i$ 成分を, $r_{1 i}, r_{2 i}$ は区 間 $[0,1]$ の一様乱数, $\lambda, c_{1}, c_{2}$ は, 各項の重み係数である。 また，

$$
\begin{array}{r}
l^{p}(k)=\underset{l}{\operatorname{argmin}}\left\{E\left(\boldsymbol{x}^{p}(l)\right) \mid l=0,1, \cdots, k\right\} \cdots(3 \mathrm{a}) \\
\left(Q(k), l^{o}(k)\right)=\underset{(q, l)}{\operatorname{argmin}}\left\{E \left(\boldsymbol{x}^{q}(l) \mid q=1,2, \cdots, P,\right.\right. \\
\quad l=0,1, \cdots, k\} \cdots(3 \mathrm{~b})
\end{array}
$$

であり, $l^{p}(k)$ は，個体 $p$ が時刻 $k$ までに関数 $E$ の值を 最も小さくした時刻を表し, そのときの位置 $\boldsymbol{x}^{p}\left(l^{p}(k)\right)$ は pbest とよばれている。また， $\left(Q(k), l^{\circ}(k)\right)$ は, $P$ 個の個 体からなる個体群の中で時刻 $k$ までに，関数 $E$ の值を最 も小さくした個体番号とその時刻を表し，そのときの位置 $\boldsymbol{x}^{Q(k)}\left(l^{\circ}(k)\right)$ は gbest とよばれている。ここで，(2) 式か ら乱数項を無くし, pbest, gbestへの移流方向を表す項を それぞれ

$$
\begin{aligned}
& \boldsymbol{F}^{p}\left(\boldsymbol{x}^{p}, k\right)=c_{1}\left\{\boldsymbol{x}^{p}\left(l^{p}(k)\right)-\boldsymbol{x}^{p}\right\} \cdots \\
& \boldsymbol{C}\left(\boldsymbol{x}^{p}, k\right)=c_{2}\left\{\boldsymbol{x}^{Q(k)}\left(l^{o}(k)\right)-\boldsymbol{x}^{p}\right\}
\end{aligned}
$$

と㧍くと，(2) 式は，

$$
\begin{array}{r}
\boldsymbol{v}^{p}(k+1)=\lambda \boldsymbol{v}^{p}(k)+\boldsymbol{F}^{p}\left(\boldsymbol{x}^{p}(k), k\right)+\boldsymbol{C}\left(\boldsymbol{x}^{p}(k), k\right) \\
\ldots \ldots \ldots \ldots \ldots \ldots(5 \mathrm{a}) \\
\boldsymbol{x}^{p}(k+1)=\boldsymbol{x}^{p}(k)+\boldsymbol{v}^{p}(k+1) \ldots \ldots \ldots \ldots(5 \mathrm{~b}) \\
p=1,2, \cdots, P \quad k=0,1,2, \cdots
\end{array}
$$

となる。さらに(5) 式を解いて級数形式で書き表すと,

$$
\begin{aligned}
\boldsymbol{x}^{p}(k+1)=\boldsymbol{x}^{p}(k)+\sum_{l=0}^{k}\left[\lambda ^ { k - l } \left\{\boldsymbol{F}^{p}\left(\boldsymbol{x}^{p}(l), l\right)\right.\right. \\
\left.\left.+\boldsymbol{C}\left(\boldsymbol{x}^{p}(l), l\right)\right\}+\lambda^{k+1} \boldsymbol{v}^{p}(0)\right] \\
\\
\ldots \ldots \ldots \ldots \ldots \ldots \ldots \ldots \ldots \ldots
\end{aligned}
$$

となる。ただし

$$
\boldsymbol{F}^{p}\left(\boldsymbol{x}^{p}(0), 0\right)=\boldsymbol{x}^{p}(0)-\boldsymbol{x}^{p}(0)=\mathbf{0}
$$

とする。以上より, PSO の計算モデルは, 速度が等比数列 形の線形慣性系のモデルに, pbestへの移流方向と, gbest への移流方向の畳み込みを外部入力とする離散時間慣性系 モデルであることがわかる。

\section{3. 連続時間 PSO モデル}

離散時間モデルであるPSO に対し，その計算モデルの 連続化を試みると，(6) 式より

$$
\begin{aligned}
\frac{d \boldsymbol{x}^{p}(t)}{d t}= & c \int_{0}^{t} e^{-a(t-\tau)}\left[\boldsymbol{F}^{p}\left(\boldsymbol{x}^{p}(\tau), \tau\right)+\boldsymbol{C}\left(\boldsymbol{x}^{p}(\tau), \tau\right)\right] d \tau \\
& +e^{-a t} \frac{d \boldsymbol{x}^{p}(0)}{d t} \ldots \ldots \ldots \ldots \ldots \ldots \ldots(8)
\end{aligned}
$$

なるモデルを導入することができる。両辺微分すると，

$$
\frac{d^{2} \boldsymbol{x}^{p}(t)}{d t^{2}}+a \frac{d \boldsymbol{x}^{p}(t)}{d t}=c\left[\boldsymbol{F}^{p}\left(\boldsymbol{x}^{p}(t), t\right)+\boldsymbol{C}\left(\boldsymbol{x}^{p}(t), t\right)\right]
$$

となる。このモデルを，連続時間 PSO モデルと称する。こ こで, $T^{p}(t) や\left(Q(t), T^{o}(t)\right)$ を(3) 式に対応して,

$$
\begin{array}{r}
T^{p}(t)=\underset{\tau}{\operatorname{argmin}}\left\{E\left(\boldsymbol{x}^{p}(\tau)\right) \mid 0 \leq \tau \leq t\right\} \cdots(10 \mathrm{a}) \\
\left(Q(t), T^{o}(t)\right)=\underset{(q, \tau)}{\operatorname{argmin}}\left\{E\left(\boldsymbol{x}^{q}(\tau)\right) \mid q=1, \cdots, P,\right. \\
0 \leq \tau \leq t\} \cdots \cdots(10 \mathrm{~b})
\end{array}
$$

とすると, $\boldsymbol{F}^{p} や \boldsymbol{C}$ は (4) 式に対応して,

$$
\begin{aligned}
& \boldsymbol{F}^{p}\left(\boldsymbol{x}^{p}, t\right)=c_{1}\left\{\boldsymbol{x}^{p}\left(T^{p}(t)\right)-\boldsymbol{x}^{p}\right\} \\
& \boldsymbol{C}\left(\boldsymbol{x}^{p}, t\right)=c_{2}\left\{\boldsymbol{x}^{Q(t)}\left(T^{o}(t)\right)-\boldsymbol{x}^{p}\right\}
\end{aligned}
$$

である。(10a) 式の $T^{p}(t)$ は, 初期時刻 $\tau=0$ から現時刻 $\tau=t$ の連続時間区間 $[0, t]$ 上の個体 $p$ の軌道 $\boldsymbol{x}^{p}(\bullet)$ に対 して関数 $E$ を評価し，その最小值を与える時刻を意味して いる。現時刻 $\tau=t$ はこれ自体, 時間变数として時々刻々 変化するので, $E$ の最小值を与える時刻 $T^{p}$ もの $t$ の関 数として $T^{p}(t)$ と記してあることに注意されたい。また， $(10 \mathrm{~b})$ 式の $\left(Q(t), T^{o}(t)\right)$ は, 連続時間区間 $[0, t]$ 上におい て, すべて個体の軌道上で $E$ を評価し，最小値を与える個 体番号とその時刻を意味している。

\section{4. 非線形散逸項を有する PSO モデル}

最適化問題を解くための，連続系の慣性系モデル

$$
\frac{d^{2} \boldsymbol{x}(t)}{d t^{2}}+a \frac{d \boldsymbol{x}(t)}{d t}=-c \nabla E(\boldsymbol{x}(t)) \cdots
$$

に対し, その線形散逸項 $a \frac{d \boldsymbol{x}(t)}{d t}$ を非線形散逸項

$$
\boldsymbol{\xi}\left(\frac{d \boldsymbol{x}(t)}{d t}\right)=d_{1}\left\{1-d_{0} \exp \left(-\frac{\left\|\frac{d \boldsymbol{x}(t)}{d t}\right\|}{d_{2} d_{0}}\right)\right\} \frac{d \boldsymbol{x}(t)}{d t}
$$

に置き換えることで，局所解へ停留を能動的に回避する非 線形モデルが, 藤田・安田らにより提案されている ${ }^{(4)}$ 。こ の非線形抵抗は, 探索点の速度 $\frac{d x(t)}{d t}$ のノルム $\left\|\frac{d x(t)}{d t}\right\|$ が, $\left\|\frac{d \boldsymbol{x}(t)}{d t}\right\|>d_{2} d_{0} \ln d_{0}$ を满たすほど大きいときは, 正抵抗と して働き, 探索点が局所解に近づき, $\left\|\frac{d \boldsymbol{x}(t)}{d t}\right\|<d_{2} d_{0} \ln d_{0}$ を満たすほどに小さくなると, 負抵抗として働くことで, 
能動的に局所解への停留を妨げる。この非線形散逸項 (13) を，(9) 式で表される連続時間 PSO に適用すると，

$$
\begin{aligned}
& \frac{d^{2} \boldsymbol{x}^{p}(t)}{d t^{2}}+\boldsymbol{\xi}\left(\frac{d \boldsymbol{x}^{p}(t)}{d t}\right)= \\
& \quad c\left[\boldsymbol{F}^{p}\left(\boldsymbol{x}^{p}(t), t\right)+\boldsymbol{C}\left(\boldsymbol{x}^{p}(t), t\right)\right] \cdots \cdots \cdots \\
& \boldsymbol{\xi}\left(\frac{d \boldsymbol{x}^{p}(t)}{d t}\right)= \\
& d_{1}\left\{1-d_{0} \exp \left(-\frac{\left\|\frac{d \boldsymbol{x}^{p}(t)}{d t}\right\|}{d_{2} d_{0}}\right)\right\} \frac{d \boldsymbol{x}^{p}(t)}{d t}
\end{aligned}
$$

となる。ここで, $c=1$ とし, 状態変数 $\boldsymbol{v}^{p}=\frac{d \boldsymbol{x}^{p}(t)}{d t}$ とす ると,

$$
\begin{aligned}
& \frac{d \boldsymbol{v}^{p}(t)}{d t}=-d_{1}\left\{1-d_{0} \exp \left(-\frac{\left\|\boldsymbol{v}^{p}(t)\right\|}{d_{2} d_{0}}\right)\right\} \boldsymbol{v}^{p}(t) \\
& +\boldsymbol{F}^{p}\left(\boldsymbol{x}^{p}(t), t\right)+\boldsymbol{C}\left(\boldsymbol{x}^{p}(t), t\right) \cdots(15 \mathrm{a}) \\
& \frac{d \boldsymbol{x}^{p}(t)}{d t}=\boldsymbol{v}^{p}(t)
\end{aligned}
$$

となる。さらに，(15) 式を，オイラー法により離散化幅 $\Delta T=1$ で, 離散化すると,

$$
\begin{aligned}
\boldsymbol{v}^{p}(k+1)= & \boldsymbol{v}^{p}(k)-d_{1}\left\{1-d_{0} \exp \left(-\frac{\left\|\boldsymbol{v}^{p}(k)\right\|}{d_{2} d_{0}}\right)\right\} \boldsymbol{v}^{p}(k) \\
& +\boldsymbol{F}^{p}\left(\boldsymbol{x}^{p}(k), k\right)+\boldsymbol{C}\left(\boldsymbol{x}^{p}(k), k\right) \cdots \cdots(16 \mathrm{a}) \\
\boldsymbol{x}^{p}(k+1)= & \boldsymbol{x}^{p}(k)+\boldsymbol{v}^{p}(k+1) \cdots \cdots \cdots \cdots \cdots(16 \mathrm{~b})
\end{aligned}
$$

となる。ここで, $\boldsymbol{F}^{p}\left(\boldsymbol{x}^{p}(k), k\right), \boldsymbol{C}\left(\boldsymbol{x}^{p}(k), k\right)$ を(4) 式に より置き換え, 乱数の項を再び加えると,

$$
\begin{aligned}
\begin{aligned}
& v_{i}^{p}(k+1)= \lambda\left(\boldsymbol{v}^{p}(k)\right) v_{i}^{p}(k)+c_{1} r_{1 i}\left\{x_{i}^{p}\left(l^{p}(k)\right)-x_{i}^{p}(k)\right\} \\
&+c_{2} r_{2 i}\left\{x_{i}^{Q(k)}\left(l^{o}(k)\right)-x_{i}^{p}(k)\right\} \cdots(17 \mathrm{a}) \\
& x_{i}^{p}(k+1)= x_{i}^{p}(k)+v_{i}^{p}(k+1) \cdots \cdots \cdots \cdots \cdots(17 \mathrm{~b}) \\
& i=1,2, \cdots, n \quad p=1,2, \cdots, P \quad k=0,1,2, \cdots
\end{aligned} \\
\text { となる。ただし, }
\end{aligned}
$$

$$
\lambda(\boldsymbol{v})=1-d_{1}+d_{1} d_{0} \exp \left(-\frac{\|\boldsymbol{v}\|}{d_{2} d_{0}}\right)
$$

で，慣性の重み $\lambda$ が，個体速度 $\boldsymbol{v}$ に従属した非線形な関数 $\lambda(\boldsymbol{v})$ で決定するモデルとなる。このモデルを, model.1 と する。

ところで，非線形散逸項 (13) 式は,

$$
\begin{aligned}
\boldsymbol{\xi}(\boldsymbol{y}) & =d_{1}\left\{1-d_{0} \exp \left(-\frac{\|\boldsymbol{y}\|}{d_{2} d_{0}}\right)\right\} \frac{d \boldsymbol{x}(t)}{d t} \\
& =(1-\lambda(\boldsymbol{y})) \frac{d \boldsymbol{x}(t)}{d t} \ldots \ldots \ldots \ldots \ldots
\end{aligned}
$$

と一般化することができる。すなわち，あるべクトル $\boldsymbol{y}$ の ノルム $\|\boldsymbol{y}\|$ が, $\|\boldsymbol{y}\|>d_{2} d_{0} \ln d_{0}$ を満たすほど大きいと き，エネルギーが散逸するよう正抵抗をもたせ， $\|\boldsymbol{y}\|<$ $d_{2} d_{0} \ln d_{0}$ を満たすほどに小さくなると，エネルギーの提供 を受ける負性抵抗をもたせることを意味している。そこで
まず，過去の探索点の情報である pbest 情報により駆動す るという, PSO の特徵を散逸項に生かしたモデルとして, $\boldsymbol{y}=\boldsymbol{x}^{p}\left(l^{p}(k)\right)-\boldsymbol{x}^{p}$ とした

$$
\begin{aligned}
& \lambda\left(\boldsymbol{x}^{p}\left(l^{p}(k)\right)-\boldsymbol{x}^{p}(k)\right)= \\
& \quad 1-d_{1}+d_{1} d_{0} \exp \left(-\frac{\left\|\boldsymbol{x}^{p}\left(l^{p}(k)\right)-\boldsymbol{x}^{p}(k)\right\|}{d_{2} d_{0}}\right)
\end{aligned}
$$

を有するモデル

$$
\begin{aligned}
v_{i}^{p}(k+1)= & \lambda\left(\boldsymbol{x}^{p}\left(l^{p}(k)\right)-\boldsymbol{x}^{p}(k)\right) v_{i}^{p}(k) \\
& +c_{1} r_{1 i}\left\{x_{i}^{p}\left(l^{p}(k)\right)-x_{i}^{p}(k)\right\} \\
& +c_{2} r_{2 i}\left\{x_{i}^{Q(k)}\left(l^{o}(k)\right)-x_{i}^{p}(k)\right\} \\
& \ldots \ldots \ldots \ldots \ldots \ldots
\end{aligned}
$$

を考えることができる。このモデルは, pbest 方向へ駆動 されるものの, 個体と pbest との距離が小さくなりすぎる と, エネルギー供給を受けた負性抵抗により, pbestへの 漸近を回避，ないしはそれから反発する特性を有するもの といえる。

さらに同様にして, 探索個体群全体の大域的な情報によ り駆動するという, PSOの結合系としての特徴を散逸項に 生かしたモデルとして, $\boldsymbol{y}=\boldsymbol{x}^{Q(k)}\left(l^{\circ}(k)\right)-\boldsymbol{x}^{p}$ とした

$$
\begin{aligned}
& \lambda\left(\boldsymbol{x}^{Q(k)}\left(l^{o}(k)\right)-\boldsymbol{x}^{p}(k)\right)= \\
& \quad 1-d_{1}+d_{1} d_{0} \exp \left(-\frac{\left\|\boldsymbol{x}^{Q(k)}\left(l^{o}(k)\right)-\boldsymbol{x}^{p}(k)\right\|}{d_{2} d_{0}}\right)
\end{aligned}
$$

を有するモデル

$$
\begin{aligned}
v_{i}^{p}(k+1)= & \lambda\left(\boldsymbol{x}^{Q(k)}\left(l^{o}(k)\right)-\boldsymbol{x}^{p}(k)\right) v_{i}^{p}(k) \\
& +c_{1} r_{1 i}\left\{x_{i}^{p}\left(l^{p}(k)\right)-x_{i}^{p}(k)\right\} \\
& +c_{2} r_{2 i}\left\{x_{i}^{Q(k)}\left(l^{o}(k)\right)-x_{i}^{p}(k)\right\} \\
& \ldots \ldots \cdots \cdots \cdots \cdots
\end{aligned}
$$

を考えると, このモデルは, gbest 方向へ駆動されるもの の, それに近づきすぎると gbestへの漸近を回避, ないし はそれから反発する特性を有するものといえる。

(21) 式や (23) 式のモデルでは, PSO に特徵的な個体を 駆動する外部の入力情報を非線形散逸項に取り込んだモデ ルであり, 単に個体の停留性を回避する model.1 とは異な り，個体の挙動に多様な動きを与えたモデルといえる。以 下では，これらのモデルをそれぞれ model.2, model.3 と 称することにする。

\section{5. パラメータと安定性}

(18) 式の慣性の重み $\lambda(\boldsymbol{v})$ は, パラメータ $d_{0}$ により, その 值が制御される。 $d_{1}=0.5, d_{2}=0.01$ としたときの, $\lambda(v)$ 


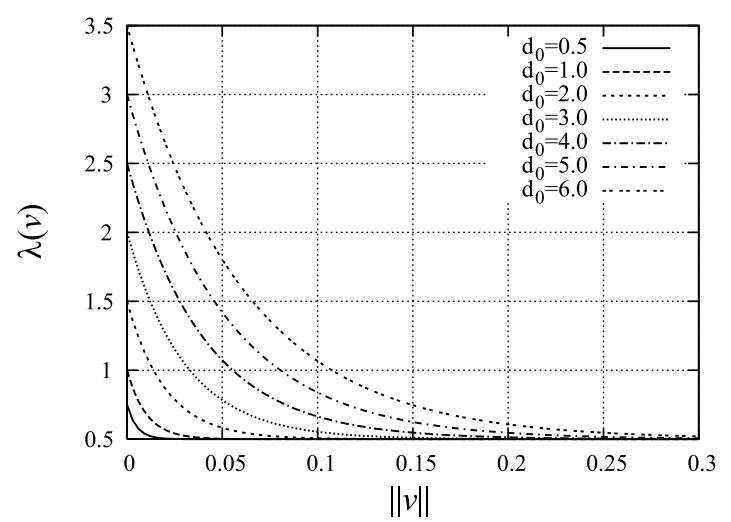

困 1 慣性の重み $\lambda$

Fig. 1. Nolinear inertia weight $\lambda$

と $\|\boldsymbol{v}\|$ の関係を図 1 に示す。一方，ランダム要素を排除し た PSO 縮約モデルのダイナミクス解析において, $c_{1}, c_{2}, \lambda$ が,

$$
\begin{aligned}
& 0<\frac{c_{1}+c_{2}}{2}<2 \lambda+2 \\
& 0 \leq \lambda<1 \ldots \ldots \ldots
\end{aligned}
$$

を満たす場合，探索点は収束傾向が強まり，満たさない場 合は発散傾向が強まることが報告されている(5)(6)。図 1 に おいて,$d_{0} \geq 1.0$ では，\|v\|が 0 に近づくにつれて $\lambda$ が 1 より大きくなって発散傾向が強まり， $d_{0}<1.0$ にのときに は, $\|\boldsymbol{v}\|=0$ においても $\lambda<1.0$ となり，収束傾向が強ま ることがわかる。そこで, $d_{0}(0)>1.0$ とし，

$$
d_{0}(k+1)=d_{0}(k)-\frac{d_{0}(0)}{K}
$$

と探索の進渉に従い徐々に小さくすることで，探索点群の 多様化と集中化を制御することができるものと期待される。 ただし， $K$ は，最大の更新回数とする。

\section{6. シミュレーション}

本章では，いくつかのベンチマーク問題に対して，提案 モデルと従来手法である PSO $(\mathrm{IWA})^{(7) \sim(9)}$ を適用し, 両者 の計算結果の比較を行った。ここで扱うベンチマークはす べて，変数の上下限值が成分に無関係に，

$$
\begin{aligned}
& \min _{\boldsymbol{x}} E(\boldsymbol{x}) \cdots \ldots \ldots \ldots \ldots \ldots \ldots \ldots \\
& \text { subj.to } b_{1} \leq x_{i} \leq b_{2}, \quad i=1, \cdots, n \cdots \cdots
\end{aligned}
$$

の形で書けるものである。すべての問題に対して，提案モ デルのパラメータを $, d_{1}=0.5, d_{2}=0.01\left(b_{2}-b_{1}\right)$ と設定 した。PSO(IWA) は，(2) 式の慣性の重み $\lambda$ を, $0.9 \rightarrow 0.4$ と線形に減少させた。また，従来モデル，提案モデルとも に, $r_{1 i}$ や $r_{2 i}$ に一様乱数を用いる場合には, $c_{1}=c_{2}=2.0$ とし, $\left|v_{i}\right|$ の最大值として $v_{\max }=0.5\left(b_{2}-b_{1}\right)$ を設定し，

$$
v_{i}(k+1)=\operatorname{sgn}\left(v_{i}(k)\right) \min \left\{v_{\max },\left|v_{i}(k)\right|\right\} \cdots
$$

とした。初期位置 $x_{i}^{p}(0)$ は, 区間 $\left[b_{1}, b_{2}\right]$ の一様乱数で与
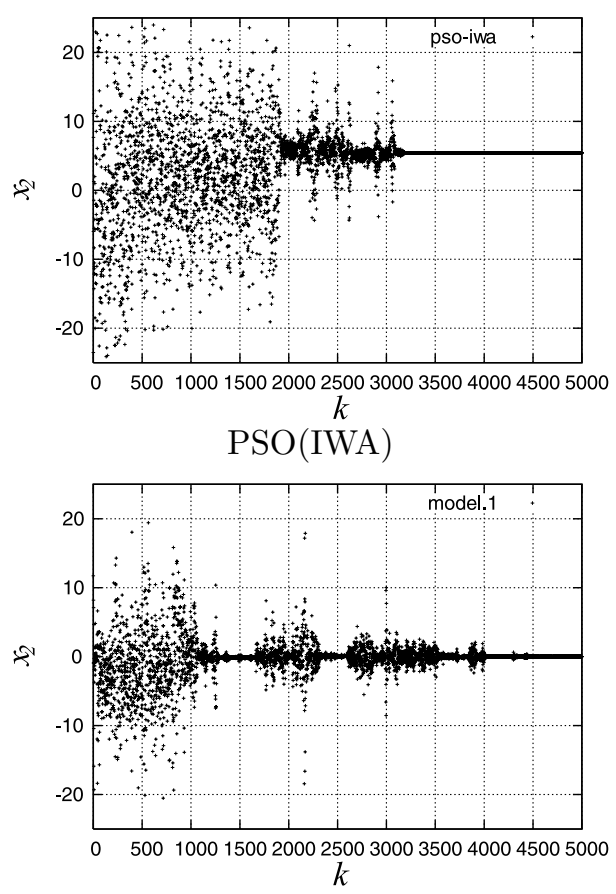

model.1

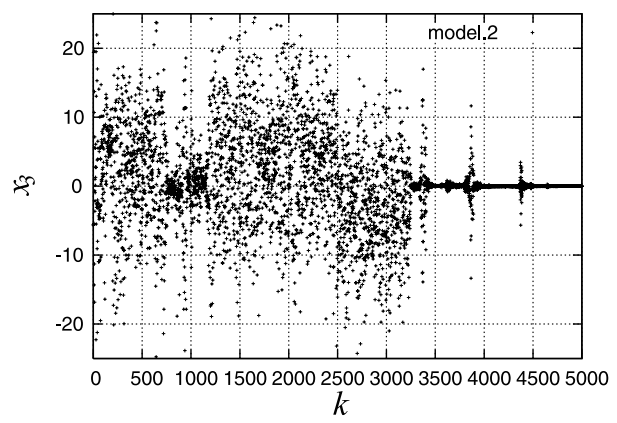

model.2

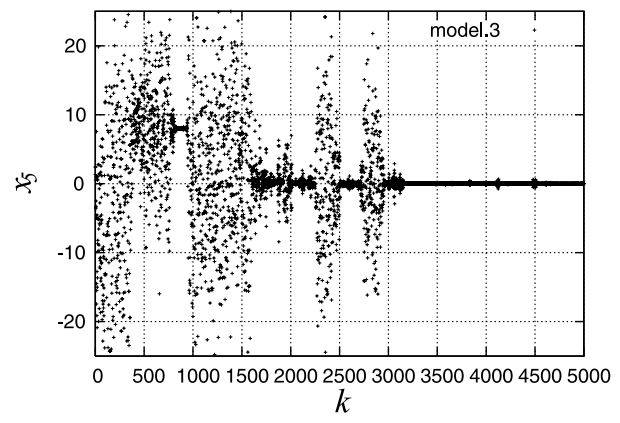

model.3

図 2 Griewank 関数最小化問題に対する探索点の軌道

Fig. 2. Time series orbit of a search point through Griewank's function $(n=6)$

え, 初速度 $v_{i}^{p}(0)$ は, 区間 $\left[-0.5\left(b_{2}-b_{1}\right), 0.5\left(b_{2}-b_{1}\right)\right]$ の 一様乱数で与えた。個体数 $P$ は, $P=20$ とした。また,

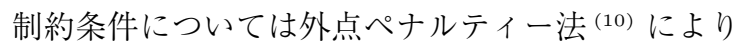

$$
\begin{aligned}
\hat{E}(\boldsymbol{x})=E(\boldsymbol{x}) & +s \sum_{i=1}^{n}\left\{\min \left(0, x_{i}-b_{1}\right)\right\}^{2} \\
& +s \sum_{i=1}^{n}\left\{\min \left(0, b_{2}-x_{i}\right)\right\}^{2}
\end{aligned}
$$

と目的関数に取り込んだ。ただ， $s$ はペナルティ係数で, 


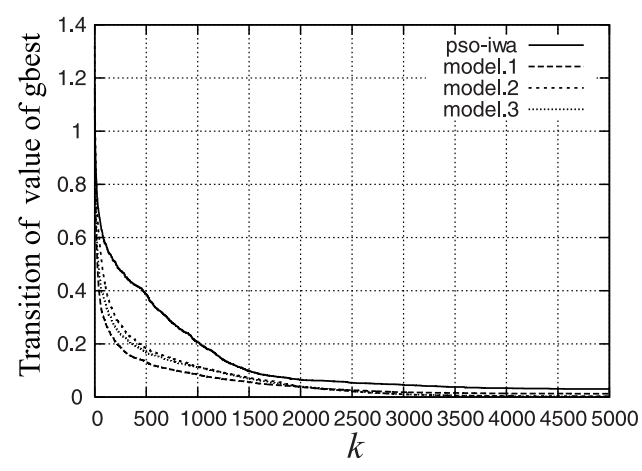

図 3 gbest の目的関数值の遷移

Fig. 3. Transition of value of gbest

$s=1.0 \times 10^{4}$ とした。最大の更新回数は $K=5000$ と した。各ベンチマーク問題に対して，100回試行した際の $\mathrm{PSO}(\mathrm{IWA})$ と各提案手法の探索性能の比較を表 1 に示す。 ただし， CR(convegence rate) は，大域的最適解への収束 を意味し, gbest と大域的最適解間のノルムが $1.0 \times 10^{-3}$ 以下となれば，収束していると判定した。 mean value, best value, worst value, $\mathrm{SD}$ (standard deviation) は, 各モデ ルにより得られた gbestの目的関数值の平均值, 最良值, 最 悪值，標準偏差を表している。CPU Time（ミリ秒）は 100 回試行のおおよその平均值である。また，提案モデルの各 $d_{0}(0)$ の設定值を表中に示した。pbest 周りの探索を回避す る model.2 と, gbest 周りの探索を回避する model.3 は従 来法と比較すると，どのベンチマーク問題に対しても良好 な大域的探索性能を有していることがわかる。なお，各モ デルの係数 $r_{1 i}$ や $r_{2 i}$ に乱数を用いずに $r_{1 i}=r_{2 i}=1$ と固 定し, pbest, gbestへの移流の重み係数を $c_{1}=c_{2}=1.0$ とした場合で，同様のシミュレーションを行った際の探索 性能の比較を表 2 に示す。乱数を用いないと探索性能が著 しく劣化しており，とくに model.3,model.2 はその劣化の 度合いが大きいことから，乱数の探索性能に与える影響が 大きいといえる。 $r_{1 i}=r_{2 i}=1, c_{1}=c_{2}=1.0$ とした根 拠は, 乱数を用いた場合の $c_{1} r_{1 i}, c_{2} r_{2 i}$ の平均值と一致さ せるためである。

ベンチマーク問題の一つである Griewank 関数最小化問 題に対して各モデルを適用した際の探索個体の一つに注目 し，その位置べクトルの 1 成分の時間発展を図 2 に示す。 なお本問題の大域的最適解は, $\boldsymbol{x}^{*}=(0, \cdots, 0)$ である。従 来法が，探索の比較的早い段階で局所解に収束しているの に対して，提案手法は，その探索軌道が収束傾向と発散傾 向を繰り返していることがわかる。また， model.1 と比較 して, model.2, model.3のほうが，この繰り返しがより大 域的に行われていることがわかる。また，同じベンチマー クに対して, gbestの目的関数值のイテレーションに対する 遷移を図 3 に示す。提案手法は，従来手法に比べ探索の初 期段階で急激に gbest の目的関数值を下げ，その後も緩や かに目的関数值を下げている。 pbest, gbestへの移流によ る探索の過程で得られた良い解周りの集中的な探索と，探
表 1 探索性能の比較

Table 1. Comparison of searching ability

\begin{tabular}{|c|c|c|c|c|c|c|c|}
\hline benchmark & nidel & $\mathrm{CR}$ & mean value & best value & wors t value & SD & CPU-Tim \\
\hline \multirow{4}{*}{$\begin{array}{c}\text { Rastrigin } \\
(\mathrm{n}=12)\end{array}$} & PSO-MK & $2 \%$ & $\begin{array}{l}.9109 \\
\end{array}$ & $\begin{array}{l}0.0 \\
\end{array}$ & $\begin{array}{l}9.94586 \\
\end{array}$ & \begin{tabular}{|l|l|l}
3.212463 \\
\end{tabular} & 310.99 \\
\hline & nødel.1 $\left(d_{0}(0)=4 \triangleright 0\right)$ & $56 \%$ & 0.606925 & 0.0 & 2.984877 & 0.591557 & \\
\hline & nødel. $2\left(d_{0}(0)=3 \bowtie 0\right)$ & $96 \%$ & 0.039798 & 0. & 0.994959 & \begin{tabular}{|l|}
0.038014 \\
\end{tabular} & 364.57 \\
\hline & nodel.3 $\left(d_{0}(0)=3 \triangleright 0\right)$ & $96 \%$ & 0.039798 & 0.0 & 0.994959 & 0.038014 & 365.33 \\
\hline \multirow{4}{*}{$\begin{array}{c}\text { Levy-No.5 } \\
(\mathrm{n}=50)\end{array}$} & PSO-IXX & $75 \%$ & 0.027089 & 0.0 & 0.370709 & 0.003283 & 2690.3 \\
\hline & nødel.1 $\left(d_{0}(0)=4 \triangleright 0\right)$ & $88 \%$ & 0.011082 & 0.0 & 0.184643 & 0.001090 & 2836.5 \\
\hline & nødel.2 $\left(d_{0}(0)=3 \triangleright 0\right)$ & $91 \%$ & 0.00431 & 0.0 & 0.061585 & 0.000247 & 2904.2 \\
\hline & nødel. $3\left(d_{0}(0)=3 \triangleright 0\right)$ & $96 \%$ & 0.00431 & 0. & 0.123114 & 0.000323 & 2908.3 \\
\hline \multirow{4}{*}{$\begin{array}{c}\text { Grie wank } \\
(\mathrm{n}=6)\end{array}$} & PSO-IMI & $39 \%$ & 0.035641 & 0.0 & 0.114410 & 0.000960 & 201.58 \\
\hline & nodel.1 $\left(d_{0}(0)=8 \triangleright 0\right)$ & $77 \%$ & 0.012826 & 0. & 0.081540 & 0.000582 & 233.73 \\
\hline & nodel.2 $\left(d_{0}(0)=8 \triangleright 0\right)$ & $91 \%$ & & 0 & .065254 & 0.000197 & 250.05 \\
\hline & nodel.3 $3\left(d_{0}(0)=8 \triangleright 0\right)$ & $95 \%$ & 0.002047 & 0.0 & 0.040495 & 0.000080 & 249.55 \\
\hline \multirow{4}{*}{$\begin{array}{c}2 \mathrm{n}-\mathrm{minima} \\
(\mathrm{n}=14)\end{array}$} & PSO-MXX & $51 \%$ & -1077 & 1096 - & -1011 & 480.511 & 268.48 \\
\hline & nodel.1 $\left(d_{0}(0)=18 \triangleright 0\right)$ & $56 \%$ & -1079 & -109 & -1011.83 & 449.175 & 8.24 \\
\hline & nodel.2 $\left(d_{0}(0)=10 \bowtie 0\right)$ & $96 \%$ & -1095.52 & 109 & -1068.38 & 30.6964 & 321.46 \\
\hline & nodel. $3\left(d_{0}(0)=10 \triangleright 0\right)$ & $92 \%$ & -1094.39 & -1096.65 & -1068.38 & 58.8349 & 319.85 \\
\hline
\end{tabular}

表 2 乱数の除いた探索性能の比較

Table 2. Comparison of searching ability without random numbers

\begin{tabular}{|c|c|c|c|c|c|c|}
\hline nchmark & nodel & $\overline{\mathrm{CR}}$ & ean value & best value & wors t value & SD \\
\hline \multirow{3}{*}{$\begin{array}{c}\begin{array}{c}\text { Rastrigin } \\
(\mathrm{n}=12)\end{array} \\
\text { (a) }\end{array}$} & 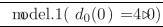 & & & & & 57.893 \\
\hline & nodel.2( $d$ & $0 \%$ & & & 683 & 466 \\
\hline & nodel.3( $d_{d}$ & $0 \%$ & & $.56-56$ & 81 & 210.02136 \\
\hline \multirow{3}{*}{$\begin{array}{c}\text { Levy-No.5 } \\
(\mathrm{n}=50)\end{array}$} & $=1$ & & & & & \\
\hline & nodel.2 2 & & & & & \\
\hline & nodel. $3\left(d_{0}(0)=3 \bowtie 0\right)$ & $0 \%$ & 55.982107 & 26.89374 & 128.51763 & 283.865603 \\
\hline \multirow{3}{*}{$\begin{array}{c}\text { Grie wank } \\
(\mathrm{n}=6)\end{array}$} & & & & & & \\
\hline & no & & & & & \\
\hline & nodel.3 $\left(d_{0}(0)=8 \bowtie 0\right)$ & $2.0 \%$ & & 0.0 & 0.392491 & 0.007217 \\
\hline \multirow{3}{*}{$\begin{array}{c}2 \mathrm{n}-\text { minima } \\
(\mathrm{n}=14)\end{array}$} & del. $1\left(d_{0}(0)=1\right.$ & 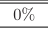 & & & & 2466.4 \\
\hline & 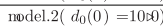 & $0 \%$ & & & & \\
\hline & nodel.3 $\left(d_{0}(0)=10 \sqsubset 0\right)$ & $0 \%$ & -932.7358 & -1068.002553 & -782.628120 & 2791.55304 \\
\hline
\end{tabular}

索初期段階で gbest, pbest 周りの探索を回避させること による多様な探索の二つをバランスよく取り入れたことが 大域的探索性能の向上につながっていると考えられる。

なお，シミュレーション環境は，CPUが Intel Pentium M 1.6GHz, OS がWindows XP SP2 で，プログラムに は $\mathrm{C}$ 言語を使用した。

\section{7. おわりに}

本論文では，PSO の慣性系を非線形化することにより， 速度がゼロとなる停留状態を防ぐことができる負性抵抗を 有する非線形散逸モデルと, 負性抵抗の項に pbest や gbest の情報を含み，それらに近接すると反発するような非線形 散逸モデルの 3 つの新しいPSO の計算モデルを導入した。 ベンチマークを通して検討した結果，提案モデルは，従来 手法と比較して, 変数次元が高く多峰性を有する目的関数 に対しても有効であることがわかった。また，速度がゼロと なるのを妨げる非線形散逸モデルよりも, pbest, gbestの 近接するときに反発する非線形散逸モデルのほうが，その 探索領域が多様化されるため, 局所解収束が妨げられ，よ り高い割合で大域的最適解を得られることがわかった。な お, 表 1 における非線形散逸項のパラメー夕 $d_{0}$ の初期值 $d_{0}(0)$ は，試行錯誤的にその最良值をもとめたものである。 これらの值が示すように，パラメータの最良の值が問題ご とに比較的大きく異なっている。このためパラメータの最 良值を問題ごとに設定する手法を開発することが今後に残 された課題といえる。

(平成 18 年 6 月 15 日受付，平成 18 年 11 月 8 日再受付) 


\section{文献}

(1) J. Kennedy and R.C. Eberhart: "Swarm Intelligence", Morgan Kaufmann Publ (2000)

(2) J. Kennedy and R.C. Eberhart: "Particle Swarm Optimization", Proc of IEEE International Conference on Neural Network (ICNN), Vol.4, pp.1942-1948 (1995)

(3) R.C. Eberhart and Y. Shi: "Particle swarm optimization: developments, applications and resources", Proc. Congress on Evolutionary Computation 2001, Seoul, Korea Piscataway, NJ: IEEE Service Center (2001)

(4) T. Fujita, K. Yasuda, and R. Yokoyama: "Global Optimization Method Using Chaos in Dissipative System", ISICE Trans., Vol.J77-A, No.6, pp.881-889 (1994) (in Japanese) 藤田・安田・横山：「散逸系カオスを用いた大域的最適化手法」, 信 学論 (A), J77-A, 6, pp.881-889 (1994)

(5) A. Ide, K. Yasuda, and N. Iwasaki: "Analysis of The Dynamics of Particle Swarm Optimization and Its Application to Global Optimization", Technical Meetingon System, SICE (2003) (in Japanese)

安田・井出・岩崎 : 「Particle Swarm Optimization：ダイナミク スの解析と大域的最適化への応用」, 第 28 回システム工学部会研究 会 (2003)

(6) K. Yasuda and N. Iwasaki: "Adaptive particle Swarm Optimization using velocity information of swarm", Proc. of IEEE Int. Conf. on Systems, Man, and Cybenetics, pp.3475-3481 (2004)

(7) R.C. Eberhart and J. Kennedy: "A New Optimizer using Particle Swarm Theory", Proceedings of the Sixth Ineternational Symposium on Micro Machine and Human Science, pp39-43 (1995)

(8) Y. Shi and R.C. Eberhart: "A modified Particle Swarm Optimizer", Proc of the 1998 IEEE conference on Evolutionary Computation, pp.69-73 (1998)

(9) R.C. Eberhart and Y. Shi: "Comparing Inertia Weights and Constriction Factors in Particle Swarm Optimization", Proc. Congress on Evolutionary Computation, pp 84-88 (2000)

(10) E. Aiyoshi and K. Shimizu: "Exercises of mathematical programming", Asakura Publishing (1985) (in Japanese) 相吉·志水:「数理計画法演習」, 朝倉書店 (1985)

(11) L.A. Rastrigin: "Systems on Extremal Control", Nauka in Russian (1994)

(12) A.V. Levy and A. Montalvo: "The Tunneling Alogorithm for the Global Minimization of Functions", SIAM J. SCI. STAT. COMPUT, Vol.6, No.1, pp.15-29 (1985)

(13) A.O. Griewank: "Generalized Descent for Global Optimization", JOTA, Vol.34, No.1, pp.15-29 (1985)

\section{付 録}

\section{1. ベンチマーク問題}

本稿で用いたベンチマークを以下に示す。Rastrigin 関 数は格子状に多数の局所的最適解を持ち，決定变数間に依 存関係を持たない。Levy-No. D 5 関数は大域的には単峰 性を有し，決定変数間に依存関係を有する。Griewank 関 数は多数の局所解を有し，決定变数間に依存関係を有する。 $2^{n}$ minima 関数は $n$ 個の決定変数に対して $2^{n}$ 個の最適解 を有し，決定変数間に依存関係を持たない。

\section{(1) Rastrign 関数 (11)}

$$
\begin{aligned}
& \min _{\boldsymbol{x}} E(\boldsymbol{x})=\sum_{i=1}^{n}\left\{x_{i}^{2}-10 \cos \left(2 \pi x_{i}\right)+10\right\} \\
& \text { subj.to }-5.0 \leq x_{i} \leq 5.0, \quad i=1, \cdots, n \\
& \boldsymbol{x}^{*}=(0.0, \cdots, 0.0), \quad E\left(\boldsymbol{x}^{*}\right)=0.0
\end{aligned}
$$

$$
\begin{gathered}
\min _{\boldsymbol{x}} E(\boldsymbol{x})=\frac{\pi}{n}\left[5.0 \sin ^{2}\left(\pi y_{1}\right)+\sum_{i=1}^{n-1}\left\{\left(y_{i}-1.0\right)^{2}\right.\right. \\
\left.\times\left(1.0+5.0 \sin ^{2}\left(\pi y_{i+1}\right)\right)\right\} \\
\left.+\left(y_{n}-1.0\right)^{2}\right] \\
y_{i}=1.0+10.0\left(x_{i}-0.25\right) \\
\text { subj.to }-1.0 \leq x_{i} \leq 1.0, \quad i=1, \cdots, n \\
\boldsymbol{x}^{*}=(0.25, \cdots, 0.25), \quad E\left(\boldsymbol{x}^{*}\right)=0.0
\end{gathered}
$$

\section{(3) Griewank 関数 ${ }^{(13)}$}

$$
\begin{aligned}
\min _{\boldsymbol{x}} E(\boldsymbol{x})= & \frac{1}{200 n} \sum_{i=1}^{n} x_{i}^{2} \\
& -\prod_{i=1}^{n} \cos \left(\frac{x_{i}}{\sqrt{i+1}}\right)+1.0
\end{aligned}
$$

subj.to $-25.0 \leq x_{i} \leq 25.0, \quad i=1, \cdots, n$

$\boldsymbol{x}^{*}=(0.0, \cdots, 0.0), \quad E\left(\boldsymbol{x}^{*}\right)=0.0$

\section{(4) $2^{n}$ minima 関数 ${ }^{(6)}$}

$$
\begin{aligned}
& \min _{\boldsymbol{x}} E(\boldsymbol{x})=\sum_{i=1}^{n}\left(x_{i}^{4}-16 x_{i}^{2}+5 x_{i}\right) \\
& \text { subj.to }-5.0 \leq x_{i} \leq 5.0, \quad i=1, \cdots, n \\
& \boldsymbol{x}^{*}=(-2.90, \cdots,-2.90), \quad E\left(\boldsymbol{x}^{*}\right)=78.319 n
\end{aligned}
$$

村 田 秀 樹 (非会員) 1979 年 8 月 26 日生。 2004 年 3 月

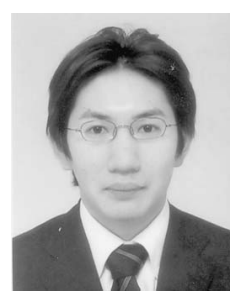
慶應義塾大学理工学部物理情報工学科卒業。同年 4 月同大学大学院理工学研究科基礎理工学専攻入 学。在学中は, 大域的最適化手法, とくに多点型 探索手法の研究を行い, 2006 年 3 月修士課程修 了。同年 4 月より，横河電機（株）に勤務。

安 田 恵一郎

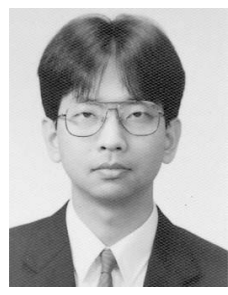

相 吉 英太郎 （正員） 1950 年 8 月 23 日生。1975 年慶應義塾

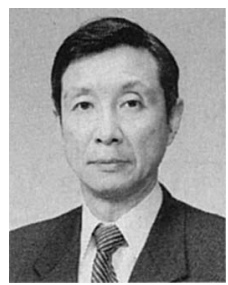

(正員) 1989 年 3 月北海道大学大学院工学研究 科電気工学専攻博士課程修了。同年 4 月東京都立 大学工学部助手, 91 年 4 月同大工学部助教授, 現 在, 首都大学東京大学院理工学研究科教授。シス テム最適化手法, ならびに電力システム工学の研 究に従事。工学博士。 大学工学部計測工学科卒業。1980 年 3 月同大学 大学院工学研究科博士課程修了。助手. 専任講師, 同理工学部助教授を経て，1996 年 4 月より同教 授。工学博士。最適化の理論と計算手法に関する 基礎研究に従事。

\section{(2) Levy-No.5 関数 (12)}

